\section{$(x)$ \\ HORIZONTES}

www.revistahorizontes.org
Horizontes. Revista de Investigación en Ciencias de la Educación Https://doi.org/10.33996/revistahorizontes.v5i17.162

Enero - marzo 2021

Volumen 5 / No. 17

ISSN: $2616-7964$

ISSN-L: $2616-7964$

pp. $108-123$

\title{
La mediación educativa como estrategia para la justicia cognitiva de comunidades y territorios de latinoamérica
}

\author{
Educational mediation as a strategy for cognitive justice in latin american communities \\ and territories
}

\section{Mediação educacional como estratégia de justiça cognitiva em comunidades e territórios latino-americanos}

ARTÍCULO DE INVESTIGACIÓN

\author{
Marco Antonio Navarrete Ávila \\ marcoantonionavarreteavila@gmail.com
}

ORCID: 0000-0002-3976-226X

\begin{abstract}
Centro de Estudios Latinoamericanos de Educación Inclusiva (CELEI), Chile
\end{abstract}
Recibido 12 de febrero 2021 | Arbitrado y aceptado 19 de marzo 2021 | Publicado en marzo 2021

\section{RESUMEN}

El objetivo de este artículo es generar un espacio de cuestionamiento crítico sobre las prácticas educativas y el rol de educadores, terapeutas, y comunidades como mediadores de aprendizajes significativos de comunidades con Diversidad Funcional Intelectual. Como estrategia metodológica se planteó una triangulación de resultados de investigación frente a nuevos referentes bibliográficos, y mediante una socialización vía digital. La inclusión de adultos con diversidad funcional en Chile y Latinoamérica es confusa y presenta incrustado un modelo obsoleto (énfasis en rehabilitación). Los mediadores del presente deben considerar la Teoría de Aprendizaje Mediado como estrategia socioeducativa para considerar la desarrollo del infinito potencial del ser humano. La investigación, y otros procesos asociados a la construcción de conocimientos sociales, requiere a su vez una actualización en sus estrategias y métodos, desde lo cual se sugiere un abordaje mediante la perspectiva de las Epistemologías del Sur.

Palabras clave: Diversidad funcional intelectual; estrategia educativa; educación inclusiva; justicia cognitiva; mediación educativa

\section{ABSTRACT}

The objective of this article is to generate a critical questioning space about educational practices and the role of educators, therapists, and communities as significant learning mediators of communities with Intellectual Functional Diversity. As a methodological strategy, a triangulation of research results was raised against new bibliographic references, and through socialization via digital. The inclusion of adults with functional diversity in Chile and Latin America is confusing and has an outdated model embedded (emphasis on rehabilitation). The mediators of the present should consider the Theory of Medium Learning as a socio-educational strategy to consider the development of the infinite potential of the human being. Research, and other processes associated with building social knowledge, in turn requires an update on its strategies and methods, suggesting an approach through the perspective of the Southern Epistemologies.

Key words: Intellectual functional diversity; educational strategy; inclusive education; cognitive justice; educational mediation 


\section{RESUMO}

O objetivo deste artigo é gerar um espaço crítico de questionamento sobre as práticas educacionais e o papel dos educadores, terapeutas e comunidades como mediadores significativos de aprendizagem de comunidades com Diversidade Funcional Intelectual. Como estratégia metodológica, foi levantada uma triangulação dos resultados da pesquisa contra novas referências bibliográficas e por meio da socialização via digital. A inclusão de adultos com diversidade funcional no Chile e na América Latina é confusa e tem um modelo ultrapassado incorporado (ênfase na reabilitação). Os mediadores do presente devem considerar a Teoria da Aprendizagem Média como estratégia socioeducativa para considerar o desenvolvimento do potencial infinito do ser humano. A pesquisa, e outros processos associados à construção do conhecimento social, por sua vez, exigem uma atualização de suas estratégias e métodos, sugerindo uma abordagem através da perspectiva das Epistemologias do Sul.

Palavras-chave: Diversidade funcional intelectual; estratégia educacional; educação inclusiva; justiça cognitiva; mediação educacional

\section{INTRODUCCIÓN}

En Chile la inclusión de adultos con Diversidad Funcional Intelectual (concepto comprendido como personas adultas que presenten diagnósticos clínicos tales como Discapacidad Intelectual -DI-/ y del Desarrollo DID-) es una problemática una vez que egresan de las Escuelas Especiales, puesto que éstos pasan a formar parte del vacío socioeducativo de la educación chilena, lo cual afecta directamente la Calidad de Vida de esta comunidad.

$\mathrm{Al}$ respecto, la legislación chilena sobre esta temática señala que, una vez cumplidos los 26 años, no existe continuidad en los apoyos socioeducativos entregados a este tipo de educación (Decreto 332 de 2012; citado en Biblioteca del Congreso Nacional de Chile, 2015, p. 3) dejando en el abandono a estos adultos una vez que egresan de sus instituciones educativas pese a sus necesidades $y$ requerimientos. A su vez, en Chile no existen estudios suficientes sobre la Calidad de Vida de estas comunidades, sin embargo, existe la investigación de Lilian Castro y colaboradoras (2015), indicando que tras aplicar la Escala de Evaluación Integral de Calidad de Vida en una población chilena institucionalizada (hogar protegido), demuestran que las personas con esta diversidad funcional suelen valorarse muy bien en estas evaluaciones, lo que implica que es un factor para considerar en la intervención de esta comunidad. Cabe destacar la escasa investigación sobre esta temática en el país.

De ello, se desprende la relevancia de esta investigación puesto que permitió dimensionar, por una parte, las implicancias de la Calidad de Vida de una población que no ha sido estudiada en profundidad en Chile, y por otra parte, considerar las barreras presentadas por esta población, principalmente en el área socioeducativa. De esta forma, se buscó implicar una perspectiva más personal e individual de la situación de vida en la cual se encuentra esta comunidad lo que, en síntesis, originó la siguiente pregunta de investigación: ¿Cuáles son las implicancias de la percepción de calidad de vida de adultos con Diversidad Funcional Intelectual, incluyendo la visión de sus cuidadoras/es principales, y cuáles son los contextos óptimos de institucionalización en un Centro Diurno Integral?

El objetivo general de esta investigación fue analizar las implicancias de la percepción de calidad de vida de adultos con Diversidad Funcional Intelectual, incluyendo la visión de sus cuidadoras/es principales, en el contexto de institucionalización en un Centro Diurno Integral, y con ello reflexionar sobre la posibilidad de inclusión social, educativa y 
política de estas comunidades una vez que son exiliados del sistema socioeducativo chileno.

A su vez, esta investigación consideró Hipótesis de trabajo de investigación, las cuales son "generales, emergentes, flexibles y contextuales, y se afinan conforme avanza la investigación" (Hernández-Sampieri et al., 2010, p. 370), la cual, puede ser "uno de los resultados del estudio" (Henderson, 2009; citado en ibíd., 2010, p. 370). Estas fueron las siguientes: a) la Calidad de Vida de adultos con Diversidad Funcional Intelectual y de sus familias se ve afectada por la falta de oportunidades para su desarrollo e Inclusión Social; b) mejorar la percepción de Calidad de Vida de sí mismos puede contribuir a desarrollar habilidades para una vida más independiente $\mathrm{y}$ autodeterminada, lo que facilitaría la Inclusión Social y una vida más digna; y c) la institucionalización de adultos con Diversidad Funcional Intelectual en un Centro Diurno Integral ofrece posibilidades para su óptimo desarrollo emocional, cognitivo y social teniendo con ello mayores oportunidades de Inclusión en la sociedad.

\section{MÉTODO}

Como diseño de esta investigación se implementó una metodología de índole cualitativa, hermenéutica, a través de la técnica investigativa del Estudio de Caso. Los datos se obtuvieron a través de Entrevistas SemiEstructuradas a los usuarios/as del Centro Diurno Integral y a sus cuidadoras/es, los que se complementaron con la información obtenida en Grupos Focales con los entrevistados/as.

Se consideró para este estudio a 12 personas (adultos con Diversidad Funcional Intelectual) institucionalizadas en un Centro Diurno Integral, de entre 31 a 63 años, que presentan diagnóstico de Discapacidad Intelectual -DI-/ y del Desarrollo -DID- de tipo Leve o Moderada, que viven en la conurbación de La Serena y Coquimbo, excluyendo a aquellos sujetos que presentan DI/DID Severa, Profunda y No Específica. Como complemento se incluyó a las/os 12 cuidadoras/es principales de los sujetos de estudio, siendo 24 personas entrevistadas en total. Además, se incluyó una descripción del ambiente y contexto de la institucionalización en un Centro Diurno Integral.

Previamente a la aplicación de acciones investigativas, se consideró tomar un consentimiento informado del sujeto entrevistado (en caso de que no pudiese responder de forma autónoma se toma un asentimiento informado), y también de su cuidador/a principal, explicando inicialmente los procesos de investigación. Luego de transcribir las grabaciones, se realizó una categorización de los Temas Ejes más mencionados en las entrevistas, todo ello según la priorización de los entrevistados/as, donde se incluyó (de forma preliminar): a) Percepción de calidad de vida de adultos con Diversidad Funcional Intelectual; b) Percepción de calidad de vida desde la visión de sus cuidadoras/es; c) Diagnóstico Médico (DI/DID Leve y Moderada); d) Institucionalización (asistencia a Centros Diurnos Integrales); y e) Dimensiones de Calidad de Vida (Autodeterminación, Derechos, Bienestar Emocional, Inclusión Social, Desarrollo Personal, Relaciones Interpersonales, Bienestar Material, y Bienestar físico) (Schalock y Verdugo, 2008).

Entonces, para iniciar este proceso se realizó se realizó un Análisis de Contenidos (Bardin, 1996) que comienza con una codificación abierta para reducir los datos encontrados a través de códigos, y luego 
estructurarla a través de una codificación axial, para finalmente, teorizar a través de una codificación selectiva. De esta forma, al seleccionar los Temas Eje a través del microanálisis de las entrevistas, se definió cada uno de estos temas de forma conceptual y operacional, dando origen a las categorías definitivas posteriormente.

En cuanto a los criterios de rigor científico, se consideró: a) credibilidad (entregando los resultados a los participantes de la investigación); b) transferibilidad (posibilidad de extender los resultados del estudio a otras poblaciones); y c) triangulación de datos (tres fuentes de información: entrevistas semiestructuradas; grupos focales; y juicio de expertos).

Finalmente, para el III Congreso Internacional de Neurociencias aplicada al desarrollo infantil integral (Centro de Investigación y Desarrollo Ecuador, CIDE) se le añadió una perspectiva de análisis desde la mirada de De Sousa Santos (2015) mediante la permeabilidad y el desarrollo de las Epistemologías del Sur Global, que infieren un prisma desde la mirada del colonizado, del oprimido y desde la Zona del No-Ser, buscando ampliar y visibilizar la polifonía de voces de estas comunidades silenciadas.

\section{RESULTADOS}

A modo de síntesis, existen bastantes temáticas que se cruzan en el análisis cualitativo de esta investigación, no obstante, la priorización de éstas solo coincide en que el Bienestar Integral es uno de los focos principales ante la calidad de vida de las personas cuidadas (Tabla 1). A su vez, se presenta una breve síntesis de los resultados dados por las Entrevistas Individuales (Tabla 2) y por los Grupos Focales (Tabla 3).

Tabla 1. Priorización de Temas Eje según codificación de los Instrumentos de Evaluación.

\begin{tabular}{llll}
\hline & Usuarios/as Institucionalizados/as & & Cuidadoras/es Principales \\
\hline 1 & Bienestar Integral & 1 & Bienestar Integral \\
2 & Relaciones Interpersonales & 2 & Derechos y Políticas Públicas \\
3 & Autodeterminación & 3 & Desarrollo Personal \\
4 & Desarrollo Personal & 4 & Relaciones Interpersonales \\
-- & Derechos y Políticas Públicas & 5 & Autodeterminación \\
\hline
\end{tabular}


Tabla 2. Síntesis de los Resultados dados por las Entrevistas Individuales

\begin{tabular}{|c|c|c|}
\hline Tema Eje & DI/DID Leve & DI/DID Moderada \\
\hline \multirow[t]{2}{*}{$\begin{array}{l}\text { Bienestar Integral } \\
\text { Incluye perspectivas } \\
\text { sobre el bienestar } \\
\text { emocional, material y } \\
\text { físico para una calidad } \\
\text { de vida digna. }\end{array}$} & $\begin{array}{l}\text { Usuarios/as Institucionalizados/as, } \\
\text { mayor énfasis en el Bienestar } \\
\text { Emocional. Impacto del 'estallido } \\
\text { social' en su Bienestar Integral. }\end{array}$ & $\begin{array}{l}\text { Usuarios/as } \\
\text { Institucionalizados/as, mayor } \\
\text { énfasis en Bienestar Emocional, ligado } \\
\text { Relaciones Interpersonales y } \\
\text { contención emocional. }\end{array}$ \\
\hline & $\begin{array}{l}\text { Cuidadoras/es, Bienestar Emocional, } \\
\text { refieren inestabilidad emocional, } \\
\text { presentando pataletas o rabietas, lo } \\
\text { cual disminuye con el desarrollo de } \\
\text { relaciones interpersonales (amigos) o } \\
\text { técnicas de relajación (jardinear, } \\
\text { pasar tiempo de ocio en solitario). La } \\
\text { sexualidad-afectividad se ve marcada } \\
\text { por el pudor y enfoques dados por la } \\
\text { televisión (romance de telenovelas). }\end{array}$ & $\begin{array}{l}\text { Cuidadoras/es, Bienestar Emocional, } \\
\text { refieren inestabilidad emocional por } \\
\text { no entendimiento o aburrimiento } \\
\text { dado por la rutina. Las mejoras } \\
\text { anímicas se relacionan con la } \\
\text { mediación emocional y actividades } \\
\text { placenteras, percibiendo la } \\
\text { afectividad de su ambiente. Se destaca } \\
\text { la enseñanza hacia la sociedad: } \\
\text { "agradecer las cosas simples de la } \\
\text { vida". }\end{array}$ \\
\hline \multirow{2}{*}{$\begin{array}{c}\text { Relaciones } \\
\text { Interpersonales } \\
\text { Incluye el desarrollo } \\
\text { de las relaciones con } \\
\text { distintas personas: } \\
\text { amistad vecindario, } \\
\text { compañeros, entre } \\
\text { otras. }\end{array}$} & $\begin{array}{l}\text { Usuarios/as Institucionalizados/as, } \\
\text { se vinculan a tres grupos: amistades } \\
\text { en la institución, grupo familiar y } \\
\text { vecindario. Se fundamentan en la } \\
\text { colaboración, ayuda y funcionalidad. }\end{array}$ & $\begin{array}{l}\text { Usuarios/as } \\
\text { Institucionalizados/as, se vinculan a } \\
\text { dos grupos: amistades en la } \\
\text { institución y grupo familiar. Se } \\
\text { fundamentan en los lazos socio- } \\
\text { afectivos y en la atención dada. }\end{array}$ \\
\hline & $\begin{array}{l}\text { Cuidadoras/es, se enfocan en el } \\
\text { desarrollo de habilidades sociales y } \\
\text { comunicacionales. Destacan la poca } \\
\text { solidez de estas relaciones. }\end{array}$ & $\begin{array}{l}\text { Cuidadoras/es, aparece temor o } \\
\text { angustia debido al bajo juicio social de } \\
\text { las personas cuidadas. El ambiente es } \\
\text { un instrumento de desarrollo integral } \\
\text { debido a las relaciones } \\
\text { interpersonales. }\end{array}$ \\
\hline
\end{tabular}




\begin{tabular}{|c|c|c|}
\hline Tema Eje & DI/DID Leve & DI/DID Moderada \\
\hline \multirow[t]{2}{*}{$\begin{array}{l}\text { Autodeterminación } \\
\text { Incluye la capacidad } \\
\text { de tomar decisiones } \\
\text { propias (vitales, } \\
\text { laborales, de ocio, } \\
\text { entre otras). }\end{array}$} & $\begin{array}{l}\text { Usuarios/as Institucionalizados/as, } \\
\text { se fundamentan en gustos propios y } \\
\text { capacidad de decidir ante situaciones } \\
\text { cotidianas. Depende de: habilidades } \\
\text { personales para la autonomía; gustos } \\
\text { personales y autoestima; relaciones } \\
\text { interpersonales y responsabilidad; y, } \\
\text { límites del ambiente y la sociedad. }\end{array}$ & $\begin{array}{l}\text { Usuarios/as Institucionalizados/as, } \\
\text { similar al grupo, pero en este caso se } \\
\text { requiere mayor guía o apoyo ante las } \\
\text { dificultades específicas de cada uno de } \\
\text { ellos. Depende de: autoimagen y } \\
\text { habilidades personales; estructura de la } \\
\text { rutina; y, desarrollo de relaciones } \\
\text { interpersonales y socialización. }\end{array}$ \\
\hline & $\begin{array}{l}\text { Cuidadoras/es, no hay vínculo entre } \\
\text { sus respuestas. No obstante, recalcan } \\
\text { el "poder de decisión", aunque con } \\
\text { límites y control del cuidador/a } \\
\text { principal. }\end{array}$ & $\begin{array}{l}\text { Cuidadoras/es, no hay vínculo entre sus } \\
\text { respuestas. Refieren a tres posturas } \\
\text { frente a la autodeterminación: libertad; } \\
\text { dependencia y anulación; y mediación. } \\
\text { Aparecen limitaciones de la comprensión } \\
\text { del lenguaje. }\end{array}$ \\
\hline \multirow{2}{*}{$\begin{array}{l}\text { Desarrollo } \\
\text { Personal } \\
\text { Incluye la } \\
\text { posibilidad de } \\
\text { aprender, adquirir y } \\
\text { tener conocimientos } \\
\text { diversos asociado a } \\
\text { la realización } \\
\text { personal. }\end{array}$} & $\begin{array}{l}\text { Usuarios/as Institucionalizados/as, } \\
\text { buscan adquirir habilidades y } \\
\text { aprendizajes por sus necesidades e } \\
\text { intereses. Temáticas de interés: } \\
\text { habilidades para la vida; desarrollo } \\
\text { socioemocional y autocontrol, y } \\
\text { habilidades de lectoescritura. }\end{array}$ & $\begin{array}{l}\text { Usuarios/as Institucionalizados/as, su } \\
\text { énfasis está en sus potencialidades, } \\
\text { intereses y necesidades intrínsecas. } \\
\text { Temáticas de interés: habilidades para la } \\
\text { vida; habilidades cognitivas; actividades } \\
\text { de ocio, recreación, artes y deportes; y } \\
\text { lectoescritura y cálculo. }\end{array}$ \\
\hline & $\begin{array}{l}\text { Cuidadoras/es, sus temáticas de } \\
\text { interés incluyen: habilidades para la } \\
\text { vida, habilidades de lectoescritura y } \\
\text { cálculo; y habilidades artísticas y } \\
\text { deportivas. }\end{array}$ & $\begin{array}{l}\text { Cuidadoras/es, refieren al concepto de } \\
\text { 'profecía autocumplida'. Sus Temáticas } \\
\text { de Interés son las mismas del grupo } \\
\text { anterior, pero suman el componente de } \\
\text { desarrollo del ocio e inclusión en la } \\
\text { comunidad. }\end{array}$ \\
\hline $\begin{array}{c}\text { Derechos y } \\
\text { Políticas Públicas }\end{array}$ & $\begin{array}{l}\text { Usuarios/as Institucionalizados/as, } \\
\text { temática no observada. }\end{array}$ & $\begin{array}{l}\text { Usuarios/as Institucionalizados/as, } \\
\text { temática no observada. }\end{array}$ \\
\hline $\begin{array}{l}\text { Incluye el respeto a } \\
\text { la diferencia, a las } \\
\text { opiniones, trato } \\
\text { igualitario, } \\
\text { intimidad y } \\
\text { confidencialidad. } \\
\text { Además, se destaca } \\
\text { la capacidad de } \\
\text { elección, conocí- } \\
\text { miento y ejercicio } \\
\text { activo de derechos. }\end{array}$ & $\begin{array}{l}\text { Cuidadoras/es, refieren a que tienen } \\
\text { los derechos de cualquier persona. } \\
\text { Detectan vulneración de derechos en } \\
\text { situaciones sociales (de índole } \\
\text { médica). Se fomenta la educación y } \\
\text { empoderamiento de la persona } \\
\text { cuidada. Temáticas críticas: cuidados } \\
\text { en el futuro y abandono social; } \\
\text { infantilización e infantilidad; y, } \\
\text { envejecimiento. }\end{array}$ & $\begin{array}{l}\text { Cuidadoras/es, se percibe sensación de } \\
\text { desesperanza ante la política pública } \\
\text { vigente por no cubrir las necesidades } \\
\text { básicas y derechos de la persona cuidada. } \\
\text { Temáticas críticas: mismas del grupo } \\
\text { anterior, pero le suman el componente } \\
\text { de Normalidad. }\end{array}$ \\
\hline
\end{tabular}

Autodeterminación Usuarios/as Institucionalizados/as, Incluye la capacidad e tomar decisiones propias (vitales, borales, de ocio, entre otras)

\section{Desarrollo}

posibilidad de prender, adquirir y

la realización personal.

\begin{abstract}
Usuarios/as Institucionalizados/as,
Cuidadoras/es, refieren a que tienen derechos de cualquier persona. enchos en sociales (de índole dica). Se fomenta la educación mpoderamiento de la persona cuidada. Temáticas críticas: cuidados en el futuro $y$ abandono social; infantilización e infantilidad; ecimiento.
\end{abstract}

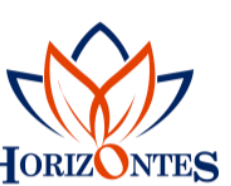


Tabla 3. Síntesis de los Resultados dados por los Grupos Focales.

\begin{tabular}{|c|c|c|}
\hline Indicador & Usuarios/as Institucionalizados/as & Cuidadoras/es \\
\hline Bienestar Integral & $\begin{array}{l}\text { No profundizado en esta } \\
\text { instancia. }\end{array}$ & $\begin{array}{l}\text { Se triangulan resultados con } \\
\text { entrevistas individuales. }\end{array}$ \\
\hline $\begin{array}{c}\text { Relaciones } \\
\text { Interpersonales }\end{array}$ & $\begin{array}{l}\text { Se triangulan resultados con } \\
\text { entrevistas individuales. }\end{array}$ & $\begin{array}{l}\text { No profundizado en esta } \\
\text { instancia. }\end{array}$ \\
\hline Autodeterminación & $\begin{array}{l}\text { Se triangulan resultados con } \\
\text { entrevistas individuales. }\end{array}$ & $\begin{array}{l}\text { Se triangulan resultados con } \\
\text { entrevistas individuales. }\end{array}$ \\
\hline Desarrollo Personal & $\begin{array}{l}\text { Se triangulan resultados con } \\
\text { entrevistas individuales. }\end{array}$ & $\begin{array}{l}\text { No profundizado en esta } \\
\text { instancia. }\end{array}$ \\
\hline $\begin{array}{l}\text { Derechos y Políticas } \\
\text { Públicas }\end{array}$ & $\begin{array}{l}\text { Temáticas } \\
\text { Infantilización e Infantilidas: } \\
\text { Envejecimiento; Respeto a la } \\
\text { Diversidad; Seguridad y Paz. }\end{array}$ & $\begin{array}{l}\text { Temáticas Críticas: Mismas del } \\
\text { grupo de personas cuidadas; } \\
\text { Futuro, Muerte y Continuidad; } \\
\text { Normalidad. }\end{array}$ \\
\hline \multirow[t]{2}{*}{ Institucionalización } & $\begin{array}{l}\text { Aspectos Positivos: sentimiento } \\
\text { personal sobre cuidados, apoyos } \\
\text { e individualidad; Desarrollo de } \\
\text { Relaciones Interpersonales e } \\
\text { Inclusión Social; Actividades } \\
\text { asociadas a requerimientos y } \\
\text { necesidades de estos sujetos. }\end{array}$ & $\begin{array}{l}\text { Aspectos Positivos: sentimiento de } \\
\text { agradecimiento y tranquilidad } \\
\text { por la institución y como eso } \\
\text { mejora su calidad de vida; Red de } \\
\text { apoyo; Oportunidad } \mathrm{de} \\
\text { desarrollo integral; Espacio de } \\
\text { aprendizaje continuo para el } \\
\text { cuidador/a. }\end{array}$ \\
\hline & $\begin{array}{l}\text { Aspectos Negativos y de } \\
\text { Transformación: } \\
\text { convivencia entre usuarios/as y } \\
\text { seguridad institucional. }\end{array}$ & $\begin{array}{l}\text { Aspectos Negativos y de } \\
\text { Transformación: equipo de planta } \\
\text { y mejora en el funcionamiento } \\
\text { institucional; Necesidad de hogar } \\
\text { protegido y/o proyectos sociales } \\
\text { para esta comunidad; Necesidad } \\
\text { de aprendizaje continuo para } \\
\text { cuidadoras/es. }\end{array}$ \\
\hline
\end{tabular}

En síntesis, se visibilizó la existencia de coincidencias y diferencias entre las percepciones de Calidad de Vida de usuarios/as institucionalizados con diagnóstico de DI/DID Leve y Moderada y sus cuidadores/as principales, presentando bastantes temáticas que dificultan su diario vivir, las cuales serán profundizadas a continuación. 
DISCUSIÓN demuestran que tan simbiótica es la

Para comenzar, sobre la propia percepción de Calidad de Vida de la persona cuidada (implicando ambas posturas), encontrando situaciones donde ambas perspectivas se alejan y difieren, como otras que son totalmente idénticas. Ello depende de la interacción entre múltiples factores, que no pueden ser abordados por un solo estudio, no obstante, los casos de mayor simbiosis se presentan cuando la cuidadora principal vive con la persona cuidada, y pasan gran parte del tiempo juntos/as, de esta manera sus posturas ante la vida se mezclan y confunden. grupos (Figura 1). Estas Zonas de Proximidad

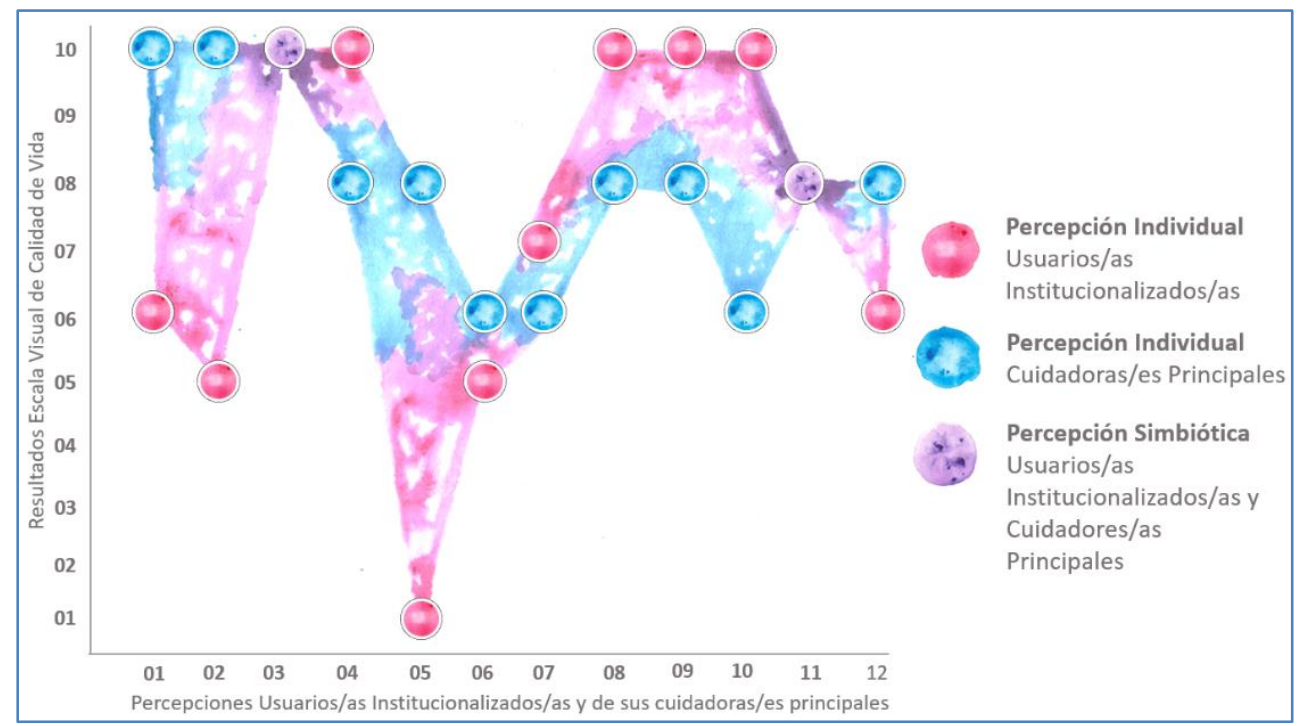

Figura 1. Zonas de proximidad y simbiosis entre percepciones de calidad de vida.

En este sentido, sobre ser adultos de la Zona del No-Ser en la sociedad contemporánea, como antecedente, el concepto de ser Niño Viejo fue acuñado por la presidenta de la institución, debido a que, a pesar de tener una edad acorde a la adultez, su actuar y necesidades son acorde a la niñez. Sin embargo, algunos de ellos están presentando tardíamente características sociales y emocionales de la adolescencia. La visión que existe sobre la Diversidad Funcional Intelectual está cargada de estigmas y discriminación, sin muchas veces comprender sus dificultades en el procesamiento cognitivo, de hecho, se puede considerar como una Diversidad Funcional Silente, ya que muchas veces visualmente no es evidente, y a su vez "no tienen la iniciativa para demostrar o explicitar sus necesidades reales" (Navarrete Ávila, 2019, p. 132). La perpetuación de estas 
perspectivas está vinculada directamente con dos conceptos: a) Infantilización, según Contino y Micheletti (2019) existe una doble infantilización en relación con estas comunidades, una vinculada con la vida del sujeto (forzamiento a permanecer en una etapa infantil, no acorde a su edad cronológica) y otra realizada por el Gobierno (Guattari y Rolnik, 2013), en donde el Estado regula y decide por los/as ciudadanos; e b) Infantilidad, en palabras de Cárdenas Palermo (2013) la noción de experiencia de la infancia "se apunta al reconocimiento de su sentido subjetivo o a la consideración de que la infantilidad de un sujeto manifiesta la relación que éste establece con los discursos y prácticas sociales tendientes a la producción de un modo genérico de ser niño" (p. 84), por lo cual esta población gracias al contexto sociocultural e histórico mantienen su infantilidad subjetiva e intersubjetiva. No obstante, se debe revalorizar el concepto de la infancia, como valor intrínseco de esta población ante situaciones de aprendizaje y motivación para alcanzar una mejor percepción Calidad de Vida, más aún, si se considera la invisibilización característica de la hegemonía de los discursos políticos, sociales y comunitarios sobre estas personas, los cuales están sesgados por las Epistemologías Occidentales Capitalistas del Norte Global (De Sousa Santos, 2015).

En cuanto a la Educación Continua y Sociocomunitaria, una de las preocupaciones inminentes de padres, madres y cuidadoras/es tras el exilio de estas personas de la educación y en gran medida de la sociedad, es qué harán una vez que egresan de estas escuelas y cómo será con ello su Calidad de Vida. En palabras de Schalock y Verdugo (2008) este concepto es "un estado de bienestar personal que es multidimensional, tiene propiedades éticas universales y émicas ligadas a la cultura, tiene componentes objetivos y subjetivos y está influenciada por factores personales $\mathrm{y}$ ambientales" (p. 22). El relacionar el concepto de Calidad de Vida a las personas que viven con este tipo de diversidad funcional ha sido de alta dificultad en el sistema educativo chileno. Estos autores postulan a cuatro prácticas profesionales en los programas de apoyo a esta población (ibíd., 2008, pp. 22-23), las cuales deben poseer: a) un acercamiento multidimensional y holístico; b) un enfoque comunitario c) el uso de buenas prácticas y de tecnología asistida; y d) el uso de apoyos individualizados. Es así como los profesionales y educadores, y por qué no nombrar a las sociedades, que continuarán apoyando a estas personas, deben considerar la Mediación, basada en la Teoría de Aprendizaje Mediado (Feuerstein et al., 1991), como estrategia educativa y social para considerar la diversidad y las singularidades múltiples que tiene cada una de las personas, en donde los usuarios/as institucionalizados/as en el Centro Diurno Integral presentan características y habilidades en la Zona de Desarrollo Real (como todas las personas), teniendo que buscar estrategias educativas mediadas, alcanzando con ello el potencial propio de cada una de estas personas, solo sí se aprovechan las oportunidades de aprendizaje de la Zona de Desarrollo Próximo, y así descubrir toda la Zona de Desarrollo Potencial de esta comunidad, principalmente con un enfoque en la dignidad y en el ejercicio de derechos.

Desde estas nuevas perspectivas socioeducativas, es que se puede dimensionar Sociedades Humanizadas a través de la Diversidad Funcional Intelectual, debido a que para humanizar la sociedad es favorable realizar un urgente cambio en la terminología que se utiliza sobre personas con diversidad 
funcional (intelectual, sensorial, o física), tanto en políticas públicas como en avisos comerciales. Según Vigotsky (1985), en su Teoría Simultánea, define que tanto el lenguaje como el pensamiento están ligados entre sí, explicando que el pensamiento y el lenguaje se desarrollan en una interrelación dialéctica y considera que las estructuras del habla se convierten en estructuras básicas del pensamiento, y sumado a ello, que la consciencia del individuo es primordialmente lingüística debido al significado que tiene el lenguaje o la actividad lingüística en la realización de las funciones psíquicas superiores del hombre. En palabras de Palacios y Romañach (2006), el peso del lenguaje es tal, "que puede acabar alienando al propio individuo al ser heredero inconsciente de modelos e ideologías" (p. 103), explicitando con ambas visiones que las palabras que se utilizan socialmente con las personas con diversidades funcionales generan contextos que pueden ser percibidos como realidades subjetivas, las cuales frecuentemente no son conscientes de su propia realidad, de su propia vulneración de derechos o de la dignidad de su propia vida. En nuestros códigos lingüísticos contemporáneos existe un sinfín de conceptos usados en diferentes contextos para denominar a las comunidades de personas con diversidad funcional. La más utilizada socialmente en Chile es discapacitado/a, lo cual definiría a una comunidad de personas sin capacidades o con menos capacidades. Es evidente que tanto en las políticas públicas de Chile sobre Inclusión Social, Educativa y Laboral se incrusta esta terminología, y se utilizan términos como: incapacitación, incapacidad, discapacidad, necesidades educativas especiales, invalidez, entre otras. Esta transformación de las palabras que se emplearán en los discursos inclusivos, al igual que la aplicación del concepto de Calidad de Vida, deben considerar a su vez, los distintos planos del sistema social que afectan la calidad de vida de las personas (Verdugo et al., 2013, p. 448), que coinciden con la teoría ecológica de Bronfenbrenner (1994), los cuales son: a) Microsistema, también conocido como Contexto Social Inmediato, tales como la familia, hogar, grupo de iguales y lugar de trabajo; b) Mesosistema, tales como vecindario, comunidad, agencias de servicios y organizaciones que afecta directamente al funcionamiento del microsistema; y c) Macrosistema, también conocido como los Patrones Culturales más amplios, tendencias sociopolíticas, sistemas económicos y otros factores relacionados con la sociedad, que afectan directamente a nuestros valores, creencias y al significado de palabras y conceptos.

Desde otra arista, sobre la muerte $y$ abandono social de esta comunidad, según Blackman (2016) estas personas pueden entender la muerte de una manera diferente debido a la carencia de información y las dificultades cognitivas, pero no se les puede negar la existencia de malestar y sufrimiento. Ante estos planteamientos, en palabras de Cristóbal Fernández y colaboradores (2018) "no recibir la noticia de la muerte puede tener consecuencias negativas en el vínculo de apego y en su autoestima, ya que puede sentirse abandonada por un ser querido que ha desaparecido de su vida sin razón aparente" (p. 58). Para evitar ello, existen evidencias relacionadas a los factores ambientales, con una sintomatología del duelo menos intensa si se les proporcionan los apoyos necesarios (Campbell y Bell, 2010). Las técnicas de intervención más utilizadas por el momento se podrían resumir en dos grandes líneas (Cristóbal Fernández et al., 2018, p. 64): a) 
espacios y grupos de contención (Boyden et al., 2010); y b) terapias específicas que complementen el proceso. Dichas intervenciones, ya sea individualmente o en grupo, requieren una planificación de acuerdo con la comprensión de la persona, los síntomas presentados y el sistema de apoyo con el que cuenta (Stoddart et al., 2002), lo cual desde la perspectiva misma de las/os cuidadoras/es se transformaría en las siguientes propuestas: a) Establecimiento de Centros Diurnos, que brinden un apoyo integral a la persona cuidada y a su entorno cercano para trabajar el proceso de transición a la vida adulta e independiente. ; y b) Creación de Hogares Protegidos, donde puedan vivir de forma digna y adecuada a las necesidades reales de la persona adulta con Diversidad Funcional Intelectual que quedaría en una eminente huerfanidad, siendo un adulto huérfano.

En este sentido, es fundamental discutir sobre los derechos y políticas públicas contemporáneas en Latinoamérica, ya que en sus sociedades se plasman a través de políticas públicas de carácter nacional e internacional con una vorágine de conceptos relacionados a la Inclusión de personas con diversidad funcional. En Chile y América Latina, estas normativas sociales, educativas y laborales están endulzadas con discursos dignificantes sobre discapacidad e igualdad de derechos, lo cual, es evidente que no se visibiliza en las comunidades con mayores requerimientos $\mathrm{y}$ necesidades socioeducativas. Las personas pertenecientes a este colectivo, en especial los adultos con Diversidad Funcional Intelectual, tienen que ver con sociedades intrínsecamente imperfectas que han establecido "un modelo de perfección al que ningún miembro concreto ha tenido acceso, y que definen la manera de ser física, sensorial o psicológicamente, y las reglas del funcionamiento social", y que éste "está relacionado con las ideas de perfección y normalidad establecidas por un amplio sector que tiene poder y por el concepto de mayorías meramente cuantitativas" (Palacios y Romañach, 2006, pp. 106-107), definiendo y delineando lo que la sociedad acepta como normal o común, lo cual se traduce en que "la negatividad del otro da paso a la positividad de lo igual" (Han, 2017, p.9), donde "la expulsión de lo distinto pone en marcha un proceso destructivo totalmente diferente: la autodestrucción" (ibíd., 2017, p. 10). Esta autodestrucción refiere a que una sociedad no inclusiva, no valora la diversidad de sus comunidades, ni siquiera admirando el enorme potencial de sus diferencias. Esta visión de la mismidad, donde no se reconocen las diferencias de las personas, está fuertemente influido a que "los macrodatos hacen superfluo el pensamiento, nos perpetúa lo igual" (Han, 2017, p. 13), donde el "imperativo neoliberal del rendimiento, atractivo y buena condición física acaba reduciendo el cuerpo a un objeto funcional que hay que optimizar" (ibíd., 2017, p.19), de esta forma se hace evidente como el sistema político y cultural perpetúa esta normalización de la población, anulando su dignidad intrínseca por completo. Los intentos de desplazar el problema completamente a la persona o completamente a la sociedad, "no han tenido demasiado éxito" (Palacios y Romañach, 2006, p. 107), por lo que es imperante transformar la sociedad y su cultura para lograr la ansiada Inclusión Auténtica, y así emancipar la dignidad y goce de derechos inherentes al ser humano de todas las personas que posean diversidades funcionales. De todos modos, se debe considerar "la resistencia del singular frente al violento poder global" (Han, 2017, p. 24), ya que el concepto de diversidad, como termino neoliberal "es un recurso que se puede explotar" (ibíd., 2017, p. 
38), lo cual describe con una visión excluyente, en donde el neoliberalismo "engendra una injusticia masiva de orden global, (...) identifica como indeseadas y excluye por tales a las personas enemigas del sistema o no aptas para él" (ibíd, 2017, p. 25), a lo que adhiere el autor que "la sociedad del consumo aspira la alteridad atópica, en favor de las diferencias consumibles heterotópicas" (p. 39), remarcando que las sociedades contemporáneas capitalistas, neoliberales y hegemónicas eliminan una visión empática sobre las diversidades funcionales, en función de plasmar la diversidad como vanidad o algo consumible.

Ante tal aclaración, la sociedad y sus jurisdicciones tienen el deber ético de diseñarse para todas las personas, sin distinción. El Diseño Universal, o Pluriversal, de la sociedad deberá empezar a percibir la discriminación intrínseca que tiene entre sus pasillos, y adoptar medidas para solucionar sus errores, utilizando el diseño para todos en un sentido amplio y complejo. La Inclusión en el siglo XXI presenta obstáculos complejos para la accesibilidad de todos en la sociedad (Figura 2) las cuales limitan la participación social de las comunidades de forma idónea. Estos obstáculos son las siguientes:

1. Políticas Públicas, el primer obstáculo refiere que, si un Estado no posee políticas públicas acordes con las diversidades funcionales, no se asegura la dignidad y ejercicio de derechos de esta vulnerada población.

2. Físicas y del Ambiente, refiere a la accesibilidad de los espacios físicos por los cual se desenvuelven las personas, con o sin diversidad funcional, resguardando siempre su seguridad física y comodidad.

3. Sensoriales y de la Comunicación, refiere a la accesibilidad sensorial y de la comunicación de los espacios físicos y sociales por los cuales se desenvuelven las personas, con o sin diversidad funcional, resguardando su comprensión y expresión del lenguaje (verbal, no verbal o alternativo) entre las personas.

4. Cognitiva e Intelectual, refiere a la accesibilidad cognitiva o intelectual de los espacios físicos y sociales por los cuales se desenvuelven las personas, con o sin diversidad funcional, resguardando el fácil entendimiento y desenvolvimiento social en los espacios públicos o privados.

5. Cultura y Paradigmas Sociales, el quinto obstáculo, y una de los más complejas de comprender, se refiere a los patrones culturales de la época temporal, cultural e histórica en la que se vive y los paradigmas sociales imbricados en sus sociedades por los cuales se desenvuelven las personas, con o sin diversidad funcional, el cual debería resguardar los derechos humanos y la dignidad de cada una de las personas. 


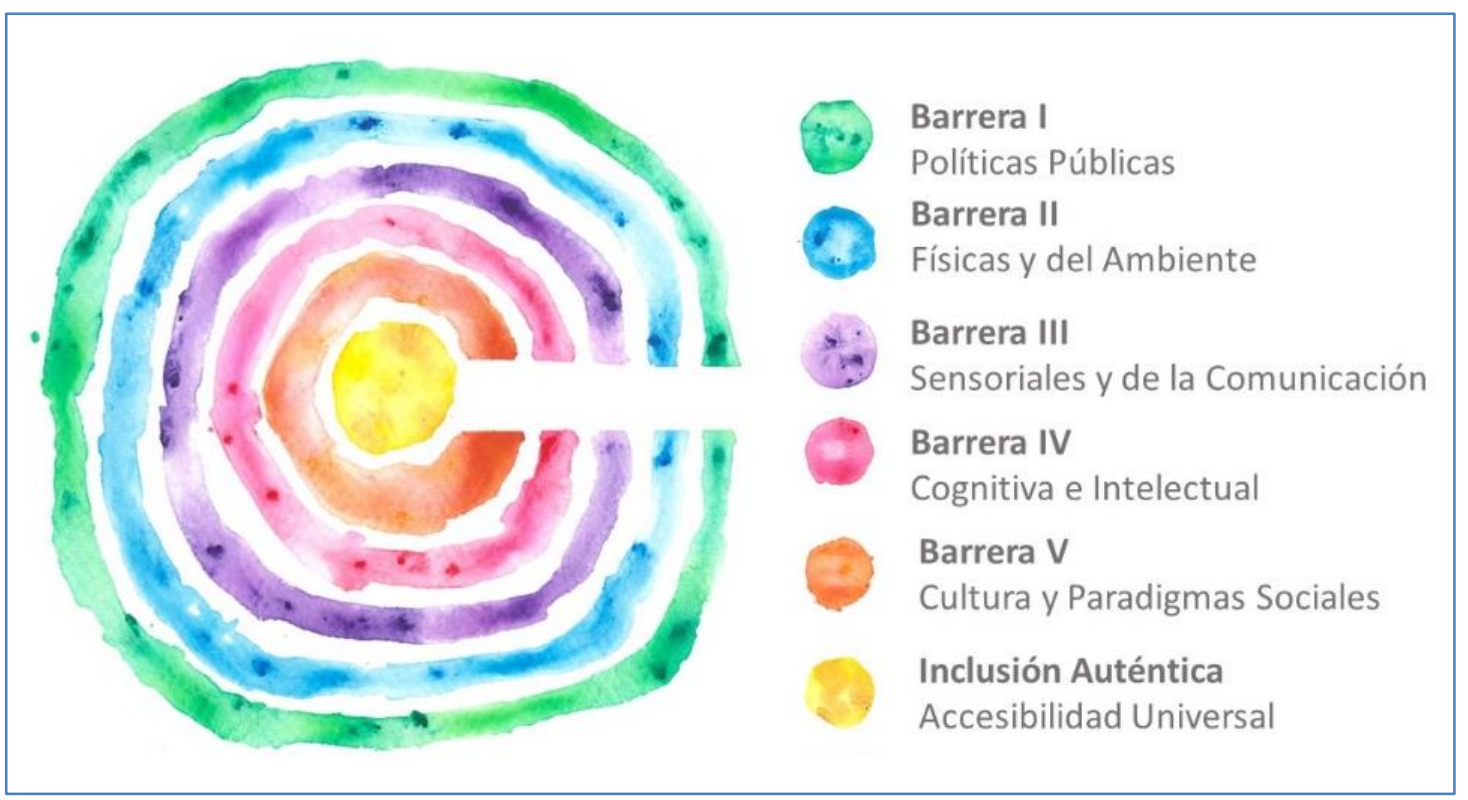

Figura 2. Obstáculos complejos de las sociedades del siglo XXI.

Para finalizar esta discusión, es imperante reflexionar sobre el concepto de funcionamiento humano, lo cual se traduce a nivel sociocomunitario en diversidades funcionales, y cómo este, es producto del cuerpo del ser humano. En la actualidad, las sociedades contemporáneas de Latinoamérica comienzan a evidenciar y comprender el racismo, el cual desde una perspectiva fanoniana (Fanon, 2010), se entiende como una jerarquía global de superioridad e inferioridad sobre la línea de lo humano, el cual produce racialización a través de "marcar cuerpos" (Grosfoguel, 2011, p. 98), lo cual patentó una fuerte tendencia histórica hacia la dominación del cuerpo en contextos educativos, sociales y políticos. De esta forma, el cuerpo racializado, concepto ecologizado desde las teorías corporales de Foucault (2002) y Le Breton (2002), y como es el caso de estos adultos de la Zona del No-Ser, tiene como trasfondo de conflicto humano la discriminación recurrente, sistemática $\mathrm{y}$ performativa dada por el modelo "social/ imperialista/ occidentalocéntrico/ capitalista/ patriarcal y moderno" (Grosfoguel, 2011, p. 98) que a través de sus sesgos eurocéntricos y contaminación epistémica permiten el status quo de las sociedades contemporáneas basadas en la dominación. De forma que, se produce la estigmatización sistemática de la fragilidad y vulnerabilidad humana, provocando finalmente la rigidez de los cuerpos, la desconexión de las emociones, la cosificación de la especie humana, y con ello, la deshumanización de las comunidades contemporáneas.

\section{CONCLUSIONES}

De esta investigación cualitativa se pueden plasmar las siguientes conclusiones que exhiben las implicancias de la percepción de Calidad de Vida de adultos de la Zona del NoSer, destacando el contexto de institucionalización de esta comunidad. La percepción de Calidad de Vida de adultos con Diversidad Funcional Intelectual está fundamentada principalmente en el Bienestar 
Integral, con mayor énfasis en el Bienestar Emocional de sí mismo. La percepción dada por su cuidador/a principal coincide en la mayor parte de las temáticas tratadas. Paradojalmente, la Calidad de Vida de los/as entrevistados/as y de sus familias se ve afectada por la vulneración de derechos como la educación o la salud, o incluso la oportunidad de vivienda, lo cual genera dificultades para su desarrollo integral e inclusión social.

Para mejorar la Calidad de Vida se percibe como prioridad el desarrollo de Habilidades Sociales y de Relaciones Interpersonales, así como el Desarrollo Personal en diversas áreas. A su vez, mejorar la percepción de Calidad de Vida de sí mismos puede contribuir a desarrollar habilidades para una vida más independiente y autodeterminada, lo que facilitaría la inclusión social y una vida más digna.

Los/as usuarios/as institucionalizados/as a pesar de no conocer realmente los conceptos de derechos y deberes en la sociedad o de políticas públicas, perciben situaciones de vulneración de derechos con énfasis en: a) respeto a la diversidad; b) infantilización e infantilidad de ellos mismos; c) envejecimiento; y d) seguridad y paz. Desde la postura de los cuidadores/as se refiere a que la persona cuidada tiene los mismos derechos que todas las personas, eso sí, con ciertas necesidades no cubiertas por el Estado, de las cuales enfatizan: a) futuro de la persona cuidada tras la muerte de su cuidador/a principal; y b) normalidad y discriminación en la sociedad.

En Chile, los apoyos socioeducativos entregados a personas con diversidades funcionales presenta un límite de edad que genera obstáculos complejos de accesibilidad e inclusión, dejando a la deriva a éstos sin justificación, sobre todo a casos de Diversidad Funcional Intelectual de carácter más severo, limitando con ello su participación social y democrática, demostrando entonces, que el Estado de Chile no se está haciendo cargo de esta comunidad. Es importante destacar que existen escasos estudios similares que aborden esta temática en el país, por lo que se hace dificultoso realizar comparaciones más empíricas con otros grupos similares.

Ante esta problemática, y todas las expuestas anteriormente, es que es fundamental considerar desarrollar instituciones públicas y/o privadas que aborden a esta población desestimada, a raíz de que gran parte de estos casos, "presentan perfiles de salud, educativo y social diferenciado, las cuales no están siendo cubiertas" (Martínez-Leal et al., 2011, p. 412). Por este motivo, es primordial diseñar programas específicos para las necesidades de estas personas, los cuales deben considerar las necesidades auténticas esta población, facilitando su autonomía, autodeterminación y participación en la sociedad contemporánea.

La institucionalización de adultos con Diversidad Funcional Intelectual en un Centro Diurno Integral ofrece posibilidades para su óptimo desarrollo emocional, cognitivo y social teniendo con ello mayores oportunidades de inclusión en la sociedad, donde se destaca el Sentimiento de Pertenencia en la institución y en la sociedad. Dentro de los aspectos que faltó profundizar en este estudio son perspectivas asociadas a la sexualidad de esta comunidad, aspectos relacionados a la condición social en la que viven, o el efecto de la tecnología en su Calidad de Vida y Alfabetización. Todos estos factores podrán ser desarrollados en investigaciones futuras.

Para concluir, es fundamental reflexionar de forma crítica sobre los conceptos de justicia 
social' y 'justicia cognitiva', ya que según De Sousa Santos (2015) no hay justicia social sin el derecho efectivo a la igualdad para vivir desde las posibilidades mentales que nos proporciona nuestra cultura, esto es, sin derecho a la justicia cognitiva, lo que refleja la carestía de oportunidades socioeducativas desde la cultura dominante, lo cual provoca e incrementa las brechas ya existentes en la grupos minoritarios de la sociedad, como lo son en este caso los adultos con Diversidad Funcional Intelectual. De esta forma, las sociedades latinoamericanas del siglo XXI deberían ayudar a construir una sociedad mejor para todas las personas, una sociedad que "acabe aceptando su propia realidad y la riqueza de su diversidad" (Palacios y Romañach, 2006, p. 188), avanzando hacia la construcción de una sociedad más humana, en que la diversidad y la diferencia de todas las personas se vean como un valor, como un potencial, "no como una carga o como una lacra en un angosto camino hacia la perfección inexistente del individuo" (ibíd., 2006, p.189).

\section{REFERENCIAS}

Bardin, L. (1996). Análisis de contenido. Madrid: Akal

Biblioteca del Congreso Nacional de Chile. (2015). Límites de edad en la Educación Especial. Departamento de Estudios, Extensión y Publicaciones. https://www.camara.cl/verDoc.aspx?prmI $\mathrm{D}=52949$ \&prmTIPO=DOCUMENTOCOMISI ON

Blackman, N. (2016). Supporting people with learning disabilities through a bereavement. Tizard Learning Disability Review, 21, 199- 202. https://doi.org/10.1108/TLDR-07-20160020

Bronfenbrenner, U. (1994). Ecological models of human development. In International
Encyclopdia of Education, 3 (2), 37- 43. Elsevier

Boyden, P., Freeman, A, y Offen, L. (2010). Setting up and running a loss and bereavement support group for adults with learning disabilities. British Journal of Learning Disabilities, 38 (1), 35-40. https://doi.org/10.1111/j.14683156.2009.00560.x

Campbell, A. y Bell, D. (2010). Sad, just sad: a woman with a learning disability experiencing bereavement. British Journal of Learning Disabilities, 39, 11-16. https://doi.org/10.1111/j.14683156.2009.00603.x

Cárdenas Palermo, Y. (2013). Experiencias de infancia (Colombia, 1930-1950): relatos del hacerse infante en las tramas de la memoria [Tesis de grado doctoral, Universidad Pedagógica Nacional de Colombia]. http://hdl.handle.net/20.500.12209/1001 4

Castro, L., Vallejos, V., Zúñiga, D., Sánchez, S., Cano, R., y Casado, M. (2015). Apoyos Educativos y calidad de vida en personas con discapacidad intelectual y del desarrollo. Proyecto Fondecyt de Iniciación $n^{\circ} 11121216$, Apoyo Convenio de desempeño UCO 1203, 1-19

Contino, A.M., Y Micheletti, A. (2019). Niñez eterna. La infantilización en la discapacidad intelectual.F@ro: revista teórica del Departamento de Ciencias de la Comunicación, 1(29), 5-23

Cristóbal Fernández, L., Alcedo Rodríguez, M., y Gómez Sánchez, L.E. (2018). Duelo en discapacidad intelectual: los avances de una década. Revista Española de Discapacidad, 5(2), 53-72. https://doi.org/10.5569/23405104.05.02.03

De Sousa Santos, B. (2015). Una Epistemología del Sur. La Reinvención del conocimiento y una emancipación social. Buenos Aires: Siglo XXI

Fanon, F. (2010). Piel Negra, Máscaras Blancas. Madrid: Akal 
Feuerstein, R., Klein, P.S., y Tannenbaum, A.J. (Eds.). (1991). Mediated learning experience (MLE): Theoretical, psychosocial and learning implications. Londres; Freund Publishing House

Foucault, M. (2002). Vigilar y Castigar. Nacimiento de la prisión. Buenos Aires: Siglo XXI

Grosfoguel, R. (2011). La descolonización del conocimiento: diálogo crítico entre la visión descolonial de Frantz Fanon y la sociología descolonial de Boaventura de Sousa Santos. Formas-Otras: Saber, nombrar, narrar, hacer, 97-108

Guattari, F., y Rolnik, S. (2013). Micropolítica. Cartografía del deseo. Buenos Aires: Tinta Limón

Han, B.C. (2017). La expulsión de lo distinto. Barcelona: Herder Editorial

Henderson, L. (2009). Qualitative research design. Londres: SAGE

Hernández-Sampieri, R., Collado-Fernández, C. y Baptista-Lucio, M.P. (2010) Metodología de la Investigación. México: McGraw Hill

Le Breton, D. (2002). La sociología del cuerpo. Buenos Aires: Nueva Visión

Martínez-Leal, R., Salvador-Carulla, L., Gutiérrez-Colosía, M.R., Nadal, M., NovellAlsina, R., Martorell, A., González-Gordón, R., Mérida-Gutiérrez, M., Ángel, S., Milagrosa-Tejonero, L., Rodríguez, A., García-Gutiérrez, J., Pérez-Vicente, A., García-Ibañez, J., y Aguilera-Inés, F. (2011). La salud en personas con discapacidad intelectual en España: estudio europeo
POMONA-II. Revista de Neurología, 53(7), 406-414.

https://www.ncbi.nlm.nih.gov/pmc/articl es/PMC3884680/

Navarrete Ávila, M. (2019). Niños Viejos: perspectivas sobre jóvenes y adultos en situación de Discapacidad Intelectual y del Desarrollo. La Serena: UNPADE La Serena. https://drive.google.com/open?id=1BGgjB ITkFebRi-AR2_sDK9-kSTJjvajJ

Palacios, A. y Romañach, J. (2006). El Modelo de la Diversidad: La Bioética y los Derechos Humanos como herramientas para alcanzar la plena dignidad en la diversidad funcional. Madrid: Diversitas

Schalock, R. y Verdugo, M.Á. (2008). Quality of life conceptual and measurement frameworks: from concept to application in the field of intellectual disabilities, Evaluation \& Program Planning, 31(2), 181190

Stoddart, K.P., Burke, L., y Temple, V. (2002). Outcome evaluation of bereavement groups for adults with intellectual disabilities. Journal of Applied Research in Intellectual Disabilities, 15(1), 28-35. https://doi.org/10.1046/j.14683148.2002.00084.x

Verdugo, M.Á., Schalock, R., Arias, B., Gómez, L., y Jordán de Urríes, F.B. (2013). Calidad de Vida. En Verdugo, M.A., Schalock, R. (Eds.) (2013). Discapacidad e Inclusión: manual para la docencia. Amarú Ediciones

Vigotsky, L. (1985). Pensamiento y lenguaje. Barcelona: Paidós 